
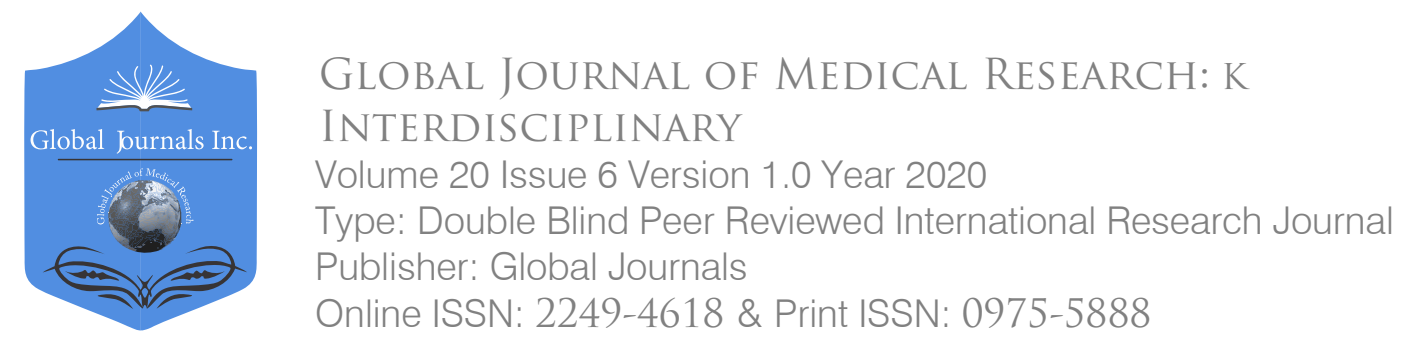

\title{
Results of the Olfactory Cognition Test Performed on 117 Peoples
}

\author{
By Naomi Katayama, Shoko Kondo, Saho Suzuki, Satoko Ishiguro, Nijiho Kondo, \\ Nana Amano \& Kaho Okuda \\ Nagoya Women's University
}

Abstract- Recently, many types of research have reported odors. There are several types of lits used for testing, but in Japan, there re odor sticks, open essences, T\&T olfactometry, etc. This time, we report that we conducted an olfactory cognitive test using open essence on healthy 117 peoples (35 males and 83 females). The Open Essence (made by FUJIFILM) has the smell as same as the odor Stick Identification Test (OSIT-J). The aromas used in the open essence includes curry, perfume, Japanese cypress, India ink, menthol, rose, wood, stinkysocks/sweat, roasted garlic, condensed milk, gas for cooking and Japanese mandarin aromas. This 12 different kinds of perception is not necessarily culture-free; the Japanese version employed. Depending on the type of odor, that were difficult to understand and some that were easy to understand. The most will-recognized odor was the smell of Curry, and the most hard to understand odor was mandarin orange. In males, the highest cognitive odor was Curry, and the lowest odor was Stir-fried garlic. In females, the highest cognitive odor was Curry, and the lowest odor was mandarin orange. In the future, it will be necessary to perform olfactory cognitive ability by age, using open essence.

Keywords: olfaction test, open essence, cognition, gender.

GJMR-K Classification: NLMC Code: W 84

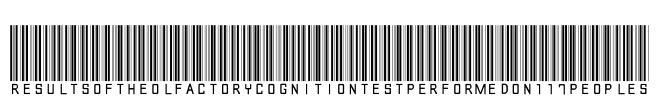

Strictly as per the compliance and regulations of:

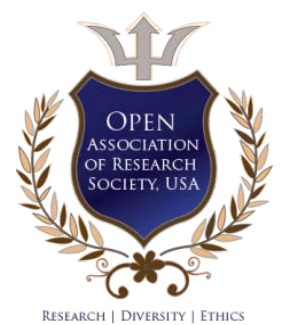

(c) 2020. Naomi Katayama, Shoko Kondo, Saho Suzuki, Satoko Ishiguro, Nijiho Kondo, Nana Amano \& Kaho Okuda. This is a research/review paper, distributed under the terms of the Creative Commons Attribution-Noncommercial 3.0 Unported License http://creativecommons.org/licenses/by-nc/3.0/), permitting all non-commercial use, distribution, and reproduction in any medium, provided the original work is properly cited. 


\title{
Results of the Olfactory Cognition Test Performed on 117 Peoples
}

\author{
Naomi Katayama ${ }^{\alpha}$, Shoko Kondo ${ }^{\circ}$, Saho Suzuki ${ }^{\rho}$, Satoko Ishiguro ${ }^{\omega}$, Nijiho Kondo ${ }^{*}$, Nana Amano ${ }^{\S}$ \\ \& Kaho Okuda ${ }^{x}$
}

Abstract- Recently, many types of research have reported odors. There are several types of lits used for testing, but in Japan, there re odor sticks, open essences, T\&T olfactometry, etc. This time, we report that we conducted an olfactory cognitive test using open essence on healthy 117 peoples (35 males and 83 females). The Open Essence (made by FUJIFILM) has the smell as same as the odor Stick Identification Test (OSIT-J). The aromas used in the open essence includes curry, perfume, Japanese cypress, India ink, menthol, rose, wood, stinkysocks/sweat, roasted garlic, condensed milk, gas for cooking and Japanese mandarin aromas. This 12 different kinds of perception is not necessarily culture-free; the Japanese version employed. Depending on the type of odor, that were difficult to understand and some that were easy to understand. The most will-recognized odor was the smell of Curry, and the most hard to understand odor was mandarin orange. In males, the highest cognitive odor was Curry, and the lowest odor was Stir-fried garlic. In females, the highest cognitive odor was Curry, and the lowest odor was mandarin orange. In the future, it will be necessary to perform olfactory cognitive ability by age, using open essence. Keywords: olfaction test, open essence, cognition, gender.

\section{INTRODUCTION}

T here are various types of olfactory recognition tests, but as a simple test, odor sticks or open essences are currently used in Japan. Olfactory disorders are classified into 1. Respiratory, 2. Peripheral, 3. Central. There are, of course, cases of age-related decline in olfactory ability. However, in recent years, it has been reported that an olfactory disorder appears an an initial symptom of Alzheimer's dementia. It is a central olfactory disorder in which the brain that processes odor information damaged. Also, the olfactory disorder is also presenting as an initial symptom in the current problem of COVID-19 infection. It is a peripheral olfactory disorder, and olfactory mucosal olfactory disorder in which the olfactory mucosa degenerates is suspected. Olfactory cognitive ability is closely related to the quality of life.

Therefore, this study focused on the perception of smell and aimed to understand the actual situation of 12 different kinds of odors closely related to Japanese life in each age and gender. To begin with, we report on the olfactory perception of 35 male and 83 female.

\section{il. Materials and Methods}

\section{a) Participants}

The participants were 35 meals and 83 females $(n=117)$ who voluntarily participated in olfactory tests. Males were 35 peoples and females were 83 peoples. Age \pm standard deviation was $29.29 \pm 16.96$ years old males and $41.89 \pm 24.66$ years old female. The maximum was 82years old, and the minimum was 12 years old male. The maximum was 87 years old, and the minimum was 16 years old female. They were healthy, not going to the hospital and taking no medication. They were self-reported and had no colds and no fever. (Table 1).

Table 1: Distrribution of age and sex (number of people)

\begin{tabular}{lcccccccc}
\hline \hline & $10 ' s$ & $20 ' s$ & $30 ' s$ & $40 ' a$ & $50 ' s$ & $60 ' s$ & $70 ' s$ & 80's \\
\hline Male $(\mathbf{n}=35)$ & 7 & 17 & 6 & 2 & 0 & 1 & 0 & 2 \\
Female (n=82) & 8 & 34 & 3 & 6 & 6 & 7 & 11 & 7 \\
Total $(\mathbf{n}=117)$ & 15 & 51 & 9 & $\mathbf{8}$ & 6 & $\mathbf{8}$ & 11 & 9 \\
\hline \hline
\end{tabular}

b) Assessment of odour identification

The Odor Stick Identification Test (OSIT-J) was used to assess odor perception for many years for our study. This test possesses high reliability and validity ${ }^{1}$ ).

Author $\alpha \rho \omega ¥ \S \chi$ : Nagoya Women’s University, Nagoya City, Japan. e-mail:naomik@nagoya-wu.ac.jp

Author a: Graduate School of Nagoya Women's University, Nagoya City, Japan.

Author o: Watanabe Hospital, Mihama town, Noma, Aichi, Japan.
The basic procedure resembles that of the San Diego Odor Identification Test ${ }^{2}$ ). The aromas used in the OSIT$\mathrm{J}$ include curry, perfume, Japanese cypress, India ink, menthol, rose, wood, stinky socks/sweat, roasted garlic, condensed milk, gas for cooking; and Japanese mandarin aromas ${ }^{3,4}$ ). This 12 different odorants perception is not necessarily culture-free, the Japanese version was employed ${ }^{3,4}$ ). Each fragrance was enclosed in microcapsules made of melamine resin ${ }^{3,4}$ ). 
In this study, we use The Open Essence (made by FUJIFILM) has the smell as same as the odor Stick Identification Test (OSIT-J). The open essence is a card type, and the scent had already applied to the card. When participants open the card, it has the same scent as OSIT-J. Each correct answer was scored as one point with the total performance score ranging from 0 to 12 points $\left.{ }^{5,6}\right)$. We defined it as follows: normal range as more than six points, borderline as 3 to 5 points, and abnormal as less than 2 points $\left.{ }^{5,6}\right)$. All of these methods are the same as in the previously reported paper ${ }^{5,6}$ ).

\section{c) Ethical review board}

This study conducted with the approval of the Ethical Review Board (Nagoya women's university Ethics Committee: 'hitowomochiitakennkyuunikansuruiinnkai'). The approval number is $30-11$.

\section{Results}

\section{a) Odour identification (number of correct answer)}

Twelve different kinds of olfactory cognitive tests conducted on female students by using the Open Essence. The results shown in Table 1. When there are six or more types of recognition among the 12 types of odors, it considered as an acceptable range (we call it a normal range). This time, 23 out of 35 males could recognize more than six kinds of odors, as same as 75 out of 83 females could recognize more than six kinds of odors. By the way, one student had four types of perceptible odors. The average value of the olfactory recognition test results of 35 males was $7.3 \pm 2.3$ and 83 females was 8.6 \pm 2.2 (Table2. and Table 3.).

Table 2: Simple olfactory cognitive results (Open essence) (number of people)

\begin{tabular}{|c|c|c|c|c|c|c|c|c|c|c|c|c|c|}
\hline & 0 & 1 & 2 & 3 & 4 & 5 & 6 & 7 & 8 & 9 & 10 & 11 & 12 \\
\hline Male（n=35) & 0 & 0 & 0 & 0 & 6 & 4 & 2 & 7 & 3 & 8 & 1 & 3 & 1 \\
\hline Female $(n=82)$ & 1 & $\mathbf{0}$ & $\mathbf{0}$ & 2 & 2 & 3 & 8 & 2 & 13 & 16 & 22 & 12 & 1 \\
\hline Total $(n=117)$ & 1 & 0 & 0 & 2 & 8 & 7 & 10 & 9 & 16 & 24 & 23 & 15 & 2 \\
\hline
\end{tabular}

Table 3: Results of olfactory cognition test in female university students (Average number of recognition \pm Standard Deviation)

\begin{tabular}{lc}
\hline \hline & Numver of recognition \pm Standard Deviation \\
\hline Male $(\mathrm{n}=35)$ & $7.3 \pm 2.3$ \\
Femela $(\mathrm{n}=\mathrm{83})$ & $8.6 \pm 2.2$ \\
Total $(\mathrm{m}=117)$ & $8.2 \pm 2.3$ \\
\hline \hline
\end{tabular}

\section{b) Odour identification (percentage of each smell)}

Next, Tables4 and 5 show the results of individually examining each of the 12 odors. Curry was the smell that both ales and females showed the highest olfactory perception. The odor that males had the lowest olfactory perception was Stir-fried garlic. But, the odor that female has the lowest olfactory perception was mandarin orange. It was the smell of Wood and Cypress that both males and females had about the same olfactory cognition in both senses. However, the olfactory perception of other odors was better in females than in males. Females had a $12.7 \%$ better olfactory perception than males with India Ink. As well, females had a $20.4 \%$ better olfactory perception than males with Perfume. Females had $18.6 \%$ better olfactory perception than males with Menthol. Females had a $16.9 \%$ better olfactory perception than males with Household gas. Females had a $14.1 \%$ better olfactory perception than males with Rose. Females had a $16.1 \%$ better olfactory perception than males with Stinky socks/ Sweaty. Females had a $33.8 \%$ better olfactory perception than males with Condensed milk. There were four types of odors in males and six types in females with olfactory recognition of $70 \%$ or more. Three of them (Curry, Household gas, Stinky socks/ Sweaty) were the same for both males and females.

Table 4: Results of olfactory test for 12 different odors in 117 people using open essence (correct number of people)

\begin{tabular}{lcccccccccccc}
\hline \hline & Indea Ink & Wood & Perfume & Menthol & Mandarin orange & Curry & Household gas & Rose & Cypress & Stinky socks/Sweaty & Condensed milk & Stir-fried garlic \\
\hline Male $(\mathrm{n}=35)$ & 14 & 21 & 19 & 23 & 11 & 35 & 27 & 17 & 26 & 26 & 16 & 10 \\
Female $(\mathrm{n}=82)$ & 52 & 55 & 62 & 70 & 23 & 79 & 78 & 52 & 58 & 75 & 66 & 32 \\
Total $(\mathrm{n}=117)$ & 66 & 76 & 81 & 93 & 34 & 114 & 105 & 69 & 84 & 101 & 82 & 42 \\
\hline \hline
\end{tabular}


Table 5: Results of olfactory test for 12 different odors in 117 people using open essence (correct answer \%)

\begin{tabular}{|c|c|c|c|c|c|c|c|c|c|c|c|c|}
\hline & Indea Ink & Wood & Perfume & Menthol & Mandarin orange & Curry & Household gas & Rose & Cypress & Stinky socks/Sweaty & Condensed millk & Stir-fried garlic \\
\hline Male $(n=35)(\%)$ & 40.0 & 60.0 & 54.3 & 65.7 & 31.4 & 100.0 & 77.1 & 48.6 & 74.3 & 74.3 & 45.7 & 28.6 \\
\hline Female $(n=82)(\%)$ & 62.7 & 66.3 & 74.7 & 84.3 & 27.7 & 95.2 & 94.0 & 62.7 & 69.9 & 90.4 & 79.5 & 38.6 \\
\hline Total $(n=117)(\%)$ & 56.4 & 65.0 & 69.2 & 79.5 & 29.1 & 97.4 & 89.7 & 59.0 & 71.8 & 86.3 & 70.1 & 35.9 \\
\hline
\end{tabular}

\section{Discussion}

The male and female olfactory cognitive test results examined. Comparing the results, there was no difference between males and females, but there was a big difference when the odors examined individually. The smell of Curry, Household gas, and Stinky socks/Sweaty had higher cognitive scores in both males and females. On the other hand, Mandarin orange and Stir-fried garlic have lower cognitive scores in males and females. Regarding other odors, females had better cognitive scores than males except for the smell of Cypress. When considering the quality of life, it is necessary to be able to recognize the odor associated with the deliciousness of food (Curry, Condensed milk, etc.). Since the Curry has a lot of recovery after eating, the smell can be recognized. However, since there are few opportunities to eat condensed milk, the smell may be difficult to understand. Higher olfactory cognition scores for Household gas and Stinky socks/ Sweaty in this study indicated that healthy people could avoid dangers (gas explosions and food poisoning). However, since there was a difference in olfactory cognition scores between males and females, it is necessary to collect more data in the future to investigate the difference in sex.

\section{Conclusions}

The participants were 35 meals and 83 females $(n=117)$ who voluntarily participated in olfactory tests. The average value of the olfactory recognition test results of 35 males was $7.3 \pm 2.3$ and 83 females was 8.6 \pm 2.2 . Comparing the results, there was no difference between males and females, but there was a big difference when the odors examined individually. The smell of Curry, Household gas, and Stinky socks/Sweaty had higher cognitive scores in both males and females. On the other hand, Mandarin orange and Stir-fried garlic have lower cognitive scores in males and females. However, the olfactory perception of other odors was better in females than in males. From this result, it is necessary to examine not only the number of correct answers but also the recognition score of 12 kinds of odors individually. In the future, we would like to perform the same olfactory cognitive test on more participants and compare the differences by sex and age.

\section{Acknowledgements}

This study was supported by the research aid of Choju-iryo-kenkyu-kaihatsuhi 30-14 and the Japanese Society of Taste Technology, 2019.

\section{References Références Referencias}

1. Kobayashi M (2005). The odor Stick Identification Test for the Japanese (OSIT-J): Clinical suitability for patients suffering from olfactory disturbance. Chemical Senses, 30(Suppl 1): i216-i217.

2. Murphy C, Anderson AJ, Markinson S (1994). Psychophysical assessment of chemosensory disorders in clinical populations. In K. Kurihara, N. Suzuki, \& H. Ogawa (Eds.) Olfaction and Taste. Tokyo: Springer Verlag Tokyo. pp. 609-613

3. Kobayashi M, Reiter ER, DiNardo JL, Costanzo MR (2007). A new clinical olfactory function test: cultural influence. Arch. Otolaryngol-Head Neck Surg. 133(4): 331-336.

4. Kobayashi M, Saito S, Kobayakawa T, Deguchi Y, Costanzo RM (2006). Cross-cultural comparison of data using the Odor Stick Identification Test for Japanese (OSIT-J). Chem. Senses. 31(4): 335-342.

5. Katayama N, Kondo S, Ootake H et al (2018). Odour and Salt Taste Identification in Older Adults: Evidence from the Yakumo Study in August, 2018. Acade. J. Med. Plants 7(3) 066-071.

6. Naomi Katayama, Shoko Kondo, Satofumi Sugimoto, Tadao Yoshida, Masaaki Teranishi, Michihiko Sone3 Yasushi Fujimoto, Hironao Otake, Hirokazu Suzuki, Takafumi Nakada, Naoki Saji, Seiichi Nakata and Tsutomu Nakashima (2019. Odour and salt taste identification in older adults: Evidence from the Yakumo. Academia Journal of Medicinal Plant,8(3): 030-035 